\title{
Recombinant Soluble T4
}

National Cancer Institute

\section{Source}

National Cancer Institute. Recombinant Soluble T4. NCI Thesaurus. Code C1473.

A recombinant form of CD4 antig en with anti-HIV application. Recombinant soluble T4 antigen (rsT4) is derived from cell surface antigen CD4 lacking its transmembrane and cytoplasmic domains, thereby the agent is mobile within tissues. rsT4 binds to the envelope glycoprotein gp110 of HIV and prevents the binding of virus to CD4 positive lymphocytes, resulting in an inhibition of virus infectivity. 